\title{
Real-time sub-MeV solar neutrino spectroscopy with Borexino and best direct limit on the neutrino magnetic moment
}

\author{
David Bravo-Berguño*i, on behalf of the Borexino Collaboration** \\ INFN Sezione Milano - Via Celoria 16, A/3/C11, 20133 Milano (Italy) \\ E-mail: david.bravo@mi.infn.it
}

\begin{abstract}
The Borexino neutrino observatory has, for the first time, provided a direct, wideband, precision spectroscopic detection of all $p p$-chain neutrinos (excepting hep) and provided the best available limits for the CNO solar neutrino component, updated recently with new data and weaker constraints. In addition, notable improvements in backgrounds, thanks to increased liquid scintillator stability, were enabled by the successful installation and continued operation of the Borexino Thermal Monitoring and Management System (BTMMS) since 2015. This higher scintillator radiopurity brings renewed perspectives for significant reductions in uncertainties, lowering of limits or, under certain conditions, a direct CNO measurement. Meanwhile, Borexino has also recently attained the best model-independent upper limit for the neutrino magnetic moment $\mu_{v}$.
\end{abstract}

XIV International Conference on Heavy Quarks and Leptons (HQL2018)

May 27- June 1, 2018

Yamagata Terrsa, Yamagata,Japan

\footnotetext{
* Speaker.

${ }^{\dagger}$ Also at Universidad Autónoma de Madrid, Ciudad Universitaria de Cantoblanco, 28049 Madrid, Spain.
} 


\section{Introduction}

The Borexino liquid scintillator (LS) neutrino observatory is devoted to performing highprecision neutrino observations, and is optimized for measurements in the low energy (sub-MeV) region of the solar neutrino spectrum. Borexino has succeeded in determining all major solar neutrino flux components already with its first dataset Phase 1 (2007-10): first direct detections of $p p$ [1] (Phase 2 data used), pep [2], ${ }^{7} \mathrm{Be}$ [3], and lowest-threshold observation of ${ }^{8} \mathrm{~B}$ [4] at $3 \mathrm{MeV}$, as well as the best available limit in the CNO solar $v$ flux [4]. More recently, high-precision determinations of the aforementioned solar neutrino fluxes have been attained using new techniques and enlarged statistics from the post-LS-purification phase: Phase 2 [5] [6]. Geoneutrinos have also been measured with high significance $\left(5.9 \sigma\right.$ [7]) by Borexino, thanks to the extremely clean $\bar{v}_{e}$ channel. These results are possible thanks to the unprecedented, extremely radio-pure conditions reached in the Active Volume (AV) of the detector (down to $\left.\leq 10^{-19} \mathrm{~g}\left({ }^{239} \mathrm{U} /{ }^{232} \mathrm{Th}\right) / \mathrm{g}(\mathrm{LS})[5]\right)$ achieved thanks to a combination of ultra-clean construction and fluid-handling techniques, as well as dedicated scintillator purification campaigns [8]. Detailed detector response determination was made possible thanks to very successful internal calibration campaigns [9] which did not disturb the uniquely radio-pure environment.

Borexino, located in the Hall C of the Gran Sasso National Laboratories' (LNGS) underground facilities (3,800 m w.e.), measures solar neutrinos via their interactions with a 278 tonnes target of organic liquid scintillator (LS): pseudocumene (PC, or 1,2,4-trimethylbenzene) solvent with 1.5 g/l 2,5-diphenyloxazole (PPO) scintillating solute, contained inside a thin, 8.5m-diameter transparent spherical nylon Inner Vessel (IV). Solar neutrinos are detected by measuring the energy and position of electrons scattered by neutrino-electron elastic interactions converted into electronic signals (photoelectrons or p.e.) by 2,212 photomultipliers (PMT) mounted on a concentric 13.7 m-diameter stainless steel sphere (SSS, see Figure 1). A software-defined Fiducial Volume (FV) is established within the AV. The volume between this IV and the SSS is filled with 889 tonnes of non-scintillating "buffer" (PC+2-3 g/L dimethylphthalate DMP) acting as a radiation shield for external $\gamma_{\mathrm{S}}$ and neutrons. The SSS is immersed in a 2,100-tonne Water Tank (WT) used as a Čerenkov detector for residual cosmic $\mu^{ \pm}$, rejecting them with a $>99.992 \%$ efficiency.

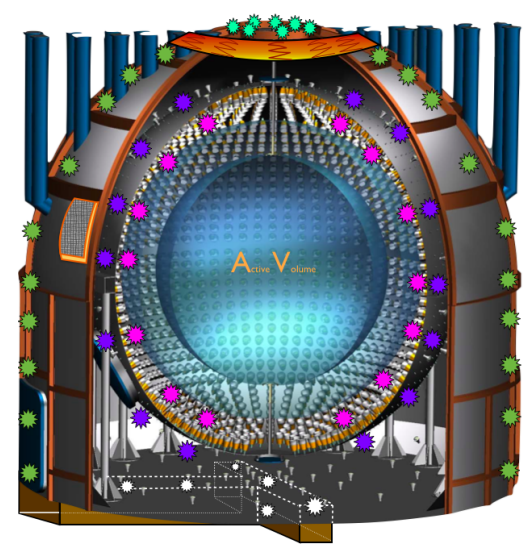

Figure 1: Cutaway of Borexino, featuring the subsystems of the Borexino Thermal Monitoring and Management System (BTMMS): the Latitudinal Temperature Probes System (LTPS, colored stars), a representative section of the Thermal Insulation System (TIS, grey-orange panel on left side of WT) and a conceptual representation of the Active Gradient Stabilization System (AGSS, top orange band). See full text for details. The Active Volume (AV) where the LS is contained is demarcated by the Inner Vessel (IV). 


\section{Borexino Phase I and II: radiopurity and analysis techniques}

Borexino's radiopurity levels have reached unprecedented levels thanks, firstly, to the ultraclean procedures employed during its construction and commissioning, and later on (2011) through the multi-step purification process [8] that brought down levels of several background species dissolved in the Active Volume. Progressive stabilization of the Inner Detector and secular decay have allowed for those levels to reach down to $\sim<10^{-19} \mathrm{~g}\left({ }^{238} \mathrm{U} / 232 \mathrm{Th} /{ }^{222} \mathrm{Rn}\right) / \mathrm{g}$. Similarly, ${ }^{210} \mathrm{Po}$ in equilibrium in the Active Volume has been reduced to near secular equilibrium levels in recent times, and ${ }^{85} \mathrm{Kr}$ is much lower than in Phase 1 data. ${ }^{210} \mathrm{Bi}$ has, however, kept slightly elevated levels. Moreover, the calibration campaign, crucial to have a precision mapping of detector response, did not introduce significant amounts of long-lived background radioisotopes.

Moreover, the Borexino Monte Carlo (MC) model ( $g 4 b x)$ was completely overhauled and upgraded during Phase 2, achieving a remarkably precise tool for understanding detector evolution: $g 4 b \times 2$ - see [14] for a detailed account of its technical characteristics and achieved precision in reproducing detector response, reaching better than $1 \%$ in the region of interest, $100 \mathrm{keV}$ to several $\mathrm{MeV}$.

Current analyses span 252 tonnes.year (71.3 fiducial tonnes and 1291.51 days, since the 11th of December 2011 -start of Phase 2 data-taking- to the 21st of May 2016), featuring optimized FV cuts (although fully consistent results are obtained with a larger FV albeit with worse uncertainties due to stronger external background $\gamma_{\mathrm{s}}$ ). After standard data quality cuts removing cosmogenic isotope activity and the remaining cosmic muon flux at the depth of the LNGS Experimental Hall C where Borexino is located $\left(\sim 1.2 \mu \mathrm{m}^{-2} \mathrm{~h}^{-1}\right)$, the remaining spectrum in the Fiducial Volume (dominated by ${ }^{14} \mathrm{C}$ and ${ }^{210} \mathrm{Po}$ peaks at low energies) can be seen in Figure 2. Additionally, radial fits are employed to characterize external backgrounds, given the approximate spherical symmetry of the detector. This approach is especially useful for the ${ }^{8} \mathrm{~B}$ analysis.

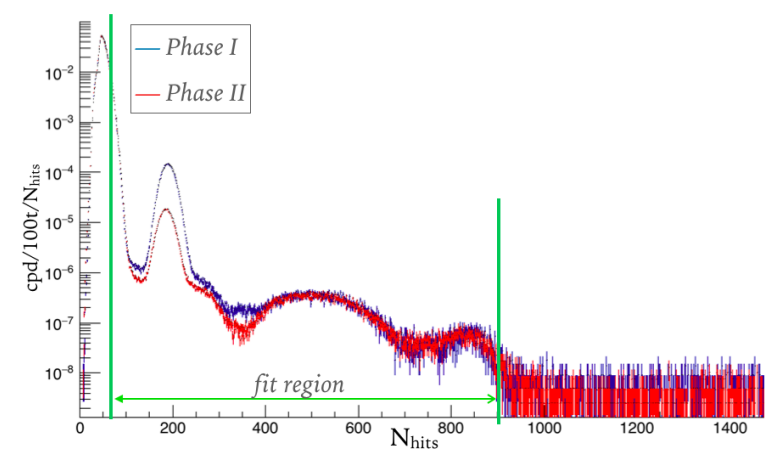

Figure 2: Borexino energy spectrum for Phase 1 and 2 data. $N_{\text {hits }}$ corresponds to $\sim 400$ at $1 \mathrm{MeV}$.

A multivariate fit between 190 and $2930 \mathrm{keV}$ is employed in order to disentangle the contribution of the different solar neutrinos to the Borexino spectrum. This allows to fit the different background components in the region of interest that have survived the standard cuts, either using constraints to its rate (for example, through the pile-up modeling developed previously for the $p p$ neutrino analysis [1]) or leaving them completely free to vary. Additionally, particle identification through pulse shape discrimination (PSD) techniques are employed, applying both $\alpha-\beta$ discrim- 
ination (through a Multi-Layer Perceptron (MLP) neutral network (NN) approach) and statistical subtraction of the constant, irreducible $\sim 30 \mathrm{cpd} / 100 \mathrm{t}$ rate of ${ }^{11} \mathrm{C}$ events (through a Three-Fold Coincidence (TFC) approach):

- MLP NN $\alpha-\beta$ PSD: The neural network utilizes 13 input variables, individually computed for each event thanks to its particular pulse time distribution. The MLP approach provides excellent PSD with minimal inefficiencies: with the MLP parameter threshold set at 0.9 to retain $\beta$ s, the $\alpha$ rejection efficiency is $>99.98 \%$ for ${ }^{214} \mathrm{Po} .{ }^{214} \mathrm{Bi}-\mathrm{Po}\left({ }^{212} \mathrm{Bi}-\mathrm{Po}\right)$ fast coincidences are thus tagged and used to suppress ${ }^{238} \mathrm{U}\left({ }^{232} \mathrm{Th}\right)$-chain daughters. Likewise, the prominent ${ }^{210}$ Po peak that survives after the standard quality cuts is largely eliminated from the final sample.

- TFC: Exploiting the spatiotemporal correlation that exists between the progenitor muon passing through Borexino's scintillator, generating neutrons and ${ }^{11} \mathrm{C}$, both providing capture or decay signals respectively, the TFC techniques achieves an efficient depletion in ${ }^{11} \mathrm{C}$ in the spectrum, albeit at a reduced exposure. For this reason, TFC-subtracted and TFC-tagged complementary spectra are used in the analysis in order to fit them simultaneously, together with the spatial distributions and the PSD variable - thereby maximizing stability and confidence in the final fit results. Furthermore, the TFC technique is further enhanced thanks to a PSD variable based on the likelihood of the position reconstruction, that allows to distinguish between $\beta^{+}$and $\beta^{-}$events (due to the positrons producing annihilation $\gamma$-rays which more widely distribute the particle's energy within the detector, and having a $\sim 50 \%$ probability of forming orthopositronium, both of which effects translate into a small but measurable delay in scintillation response widening the pulse shapes from $\beta^{+}$s).

Finally, two approaches to the fit are employed: analytical and Monte Carlo. The former approach has better adaptation capability to possible unknown detector response function systematics, and can convolve the spectral shapes, but on the other hand is more sensitive to correlations and parameter lockup in unphysical values ("railings"). The latter approach, being tuned through calibration data, automatically takes into account the true detector response, but presents larger challenges when modeling systematics and needs a large, computationally-intensive set of statistics to achieve high precision. Together, both approaches are useful to constrain systematic errors.

Special treatment of ${ }^{208} \mathrm{Tl}$ background events in the energy region where most of the ${ }^{8} \mathrm{~B}$ events lie call for a slightly modified fiducial volume and data analysis approach. This energy region (High Energy Region, or HER) is further subdivided into HER-I and HER-II in order to utilize the maximum volume possible depending on where backgrounds lie. HER-I utilizes no radial cut, and just a $z<+2.5 \mathrm{~m}$ selection cut in order to avoid external contamination seeping into the AV through a small leak on the top part of the Inner Vessel, from the less radiopure Inner Buffer. On the other hand, HER-II uses as FV the whole AV, since no remaining background is identified for this energy region. Both high-energy analyses employ most of Phase 1 data together with the lowenergy Phase 2 dataset, for a total of more than 11 times the previous exposure. As mentioned above, radial distributions are fitted in order to better constrain the spatially-uniformly distributed ${ }^{8} \mathrm{~B}$ signal in both HER windows from the radially-dependent background event distributions. Better 
constrained systematics allow the analysis to account for backgrounds that were previously not considered individually (such as neutron capture on $\mathrm{Fe}$ and $\mathrm{C}$, or ${ }^{11} \mathrm{Be}$ ).

\section{Simultaneous solar spectroscopy of pp, pep, ${ }^{7} \mathrm{Be}$ and ${ }^{8} \mathrm{~B}$}

Fits in the Low Energy Region (LER) do not involve ${ }^{8} \mathrm{~B}$ in a significant way, so its rate determined in the HER analysis is fixed in the fit. CNO neutrino rates are fixed to the ones predicted by the high- and low-metallicity (HZ and LZ) SSMs, since CNO rates cannot yet be disentangled directly as discussed in the upcoming Section 4. The differences between SSM predictions only affect in any meaningful way the pep rate, which is therefore reported as two separate results. Regardless of the particular value, this study represents the first time the pep flux is observed at more than $5 \sigma$. A CNO upper limit, representing a comparable result to the one published in the previous ${ }^{8} \mathrm{~B}$ analysis but more robust due to the weaker assumptions employed, was also derived using the LER analysis and placing a constraint on the ratio between the $p p$ and $p e p$ rates (which yields identical results for the upper limit when considering both HZ and LZ SSMs). A competitive, though not world-best, counting-based hep neutrino flux upper limit is also determined for the first time in Borexino, which means the present study covers all solar neutrino components. Fits in the High Energy Regions (HER-I and -II) yield compatible results which are combined into one single HER flux.

The ratio of ${ }^{7} \mathrm{Be}$ to ${ }^{8} \mathrm{~B}$ fluxes provide information toward a high- or low-metallicity SSM, with smaller uncertainties in Borexino's measurements than the $1 \sigma$ interval given by theoretical models, although only at a mild significance level which decreases when Borexino's results are combined with those available from other experiments. Additionally, the survival probability for each of the neutrino species is determined for this species' average energy, showing an excellent agreement with the MSW-LMA solution for matter-enhanced oscillations.

\section{BTTMS: relation between background and thermal stabilities in Borexino}

The unprecedented radiopurity levels reached in Borexino's LS are the key to the uniqueness of the detector's results. The conditions reached after the purification campaign in 2011 [5] mean that mixing of the free scintillating fluid inside the IV could cause unwanted background fluctuations that, with careful management measures, may be minimized or avoided.

Of particular importance to Borexino's future is the effort toward measuring the sub-leading, but crucial, CNO solar neutrino component ( $\leq 1 \%$ of the Sun's output [10]). Its neutrino recoil spectral shape (endpoint at $E_{\max }=1.74 \mathrm{MeV}$ ) places it under several intrinsic Borexino backgrounds, in particular ${ }^{85} \mathrm{Kr}$ and ${ }^{210} \mathrm{Bi}$, whose spectral shapes exhibit a large degree of correlation with the solar neutrino signal -especially so for ${ }^{210} \mathrm{Bi}$ in the $\sim 400$ p.e. energy window, where the $\mathrm{CNO}$ rate is briefly expected to be higher than the neighboring, ${ }^{7} \mathrm{Be}$ and pep neutrinos . It is estimated a $\leqslant 10 \%$ precision in the determination of the ${ }^{210} \mathrm{Bi}$ concentration in Borexino's $\mathrm{FV}$ is needed, during a long enough time period, to collect the very low expected CNO $v$ counts ( $\sim 3-5 \mathrm{cpd} / 100$ tonnes).

Its decay daughter ${ }^{210} \mathrm{Po}$ provides an accurate method for succeeding in this determination. Indeed, ${ }^{210} \mathrm{Po}$ 's $\alpha$ decay allows for it to be efficiently tagged out through Pulse-Shape Discrimination (PSD) techniques with very low inefficiencies. Conversely, the $\beta^{-}$decay of bismuth $(\mathrm{Q}=1160 \mathrm{keV}$, 
$t_{1 / 2}=5$ days) provides an indistinguishable (only statistically-subtractable) signal to $v_{e}-e^{-}$elastic scatterings which cannot determine the rate down to the required uncertainty levels, due to the shape degeneracy between the bismuth and solar $v$ components. Indeed, once legacy out-of-equilibrium ${ }^{210}$ Po levels have decayed away $\left(t_{1 / 2}=138.4\right.$ days), the decay curve would asymptotically reach a plateau baseline corresponding to the secular equilibrium levels of ${ }^{210} \mathrm{Bi}$. This condition has been close at hand for most of Borexino's Phase 2 DAQ period -but new out-of-equilibrium, regionallysignificant fluctuations in the ${ }^{210} \mathrm{Po}$ levels have prevented reaching it (see Figure 3).

Crucially, it is known ${ }^{210} \mathrm{~Pb}$ (parent of ${ }^{210} \mathrm{Bi}, t_{1 / 2}=22.3$ years, off-threshold-low Q-value) exhibits higher concentrations in the IV, and consequently provides a continuous, "inexhaustible" source of ${ }^{210} \mathrm{Bi}$-Po. Historically, ${ }^{210}$ Po fluctuations show a correlation with large environmental temperature excursions in the experimental Hall, hinting at a possible mechanism for replenishment of out-of-equilibrium polonium in the FV: fluid mixing through temperature-driven convection from the AV's periphery around the IV toward the center. Concurrently, the regional homogeneity and stability in ${ }^{210} \mathrm{Bi}$ concentration suggests that the underlying fluid-dynamics are slow enough to prevent most of this isotope to be transported inside the FV faster than it decays, establishing a soft upper limit in radial fluid velocity of $\frac{\mathscr{O}(m)}{\mathscr{O}\left(2 \cdot 10^{6}\right)} \sim<5 \cdot 10^{-7} \mathrm{~m} / \mathrm{s}$.

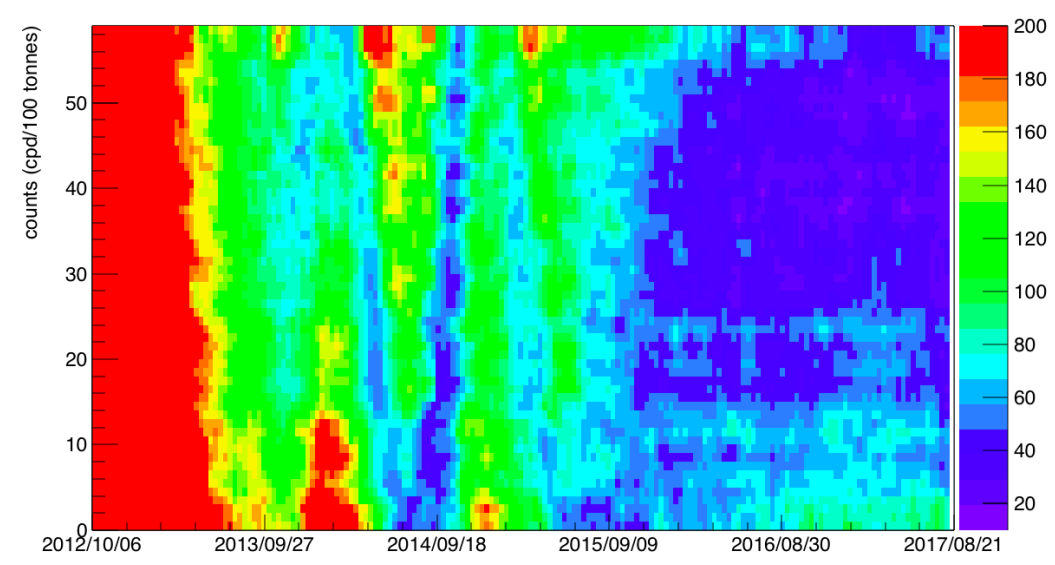

Figure 3: Regional map of historical polonium concentrations (color code: cpd/100 tonnes). Expected plateau levels due to equilibrium ${ }^{210} \mathrm{Bi}$ in the scintillator are $\sim 20 \mathrm{cpd} / 100$ tonnes. The $\mathrm{Y}$ axis indicates regional subdivisions of a $3 \mathrm{~m}$-radius $\mathrm{FV}$, running from its bottom to its top.

Deployment of the three BTMMS subsystems was completed in several periods from 20142016:

- Latitudinal Temperature Probes System (LTPS)

Sixty-three custom-calibrated, replaceable, precision temperature sensors installed throughout Borexino provide continuous readings with a finely-spaced, sub- $0.05^{\circ} \mathrm{C}$ relative precision and comparable absolute accuracy [11]. Most of them lie on the same North-South plane (hence "Latitudinal" System), which is useful to contextualize their data. The sensors are grouped in three "phases", based on their location within Borexino: Phase I comprises 28 sensors installed inside the so-called "re-entrant ports" that connect the exterior to the Outer Buffer ( $0.5 \mathrm{~m}$ inside the SSS for Phase I.a's 14 sensors and $0.5 \mathrm{~m}$ outside for I.b's), at SSS 
latitudes of approximately $\pm 65^{\circ}, \pm 50^{\circ}, \pm 26^{\circ}$ and $+7^{\circ}$; Phase II provides in-plane information on the exterior WT wall (10 sensors, II.a) as well as redundant information on different parts of the bottom heat sink (in the SOX pit, II.b); and Phase III corresponds to 7 (III.a+ III.b) sensors on the top of of the detector to monitor its warmest, most thermally-unstable area, as well as two external air monitors (III.c). They can be re-calibrated or replaced when needed thanks to their independent, extractable design. The LTPS provides continuous data since as early as November 2014 (Phase I.a) as well as input for numerical fluid-dynamical studies (see Section 5).

- Thermal Insulation System (TIS)

Fully covering Borexino's exterior WT wall and auxiliary structures since late 2015, the TIS comprises two mineral wool layers with an aluminized exterior coating $(0.3 \mathrm{~W} /(\mathrm{m} \cdot \mathrm{K}), 20$ $\mathrm{cm}$ thick) that thermally attenuate Borexino's coupling to the exterior environment, limiting thermal disturbances to the interior fluids. It is estimated that $\sim 60 \%$ of seasonal heat transfer is avoided thanks to it, and isotherm divergences from horizontal are also minimized. Phase II.a LTPS sensors are located under it. As a result of the TIS installation (and the shutdown of water circulation in the WT's bottom half), the vertical temperature gradient between Borexino's top and bottom has increased to its historical maximum, stabilizing the vertical fluid stratification to near-equilibrium (see Figure 4), which inhibits convective motions triggering LS mixing.

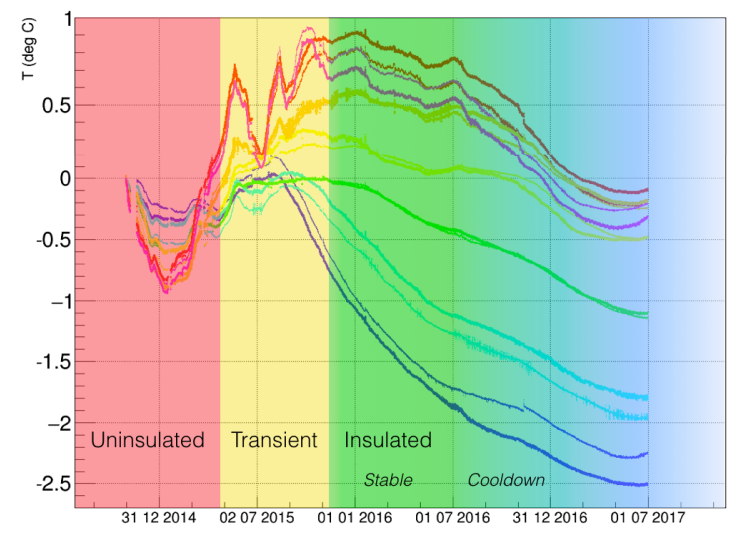

Figure 4: Relative temperature behavior in BorexinoÕs OB from the Phase I.a LTPS, normalized to initial temperature. TIS influence is evident, already since its initial deployment in mid-2015.

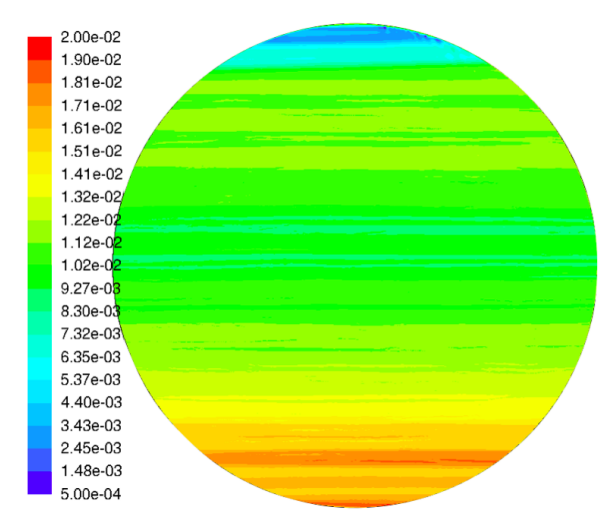

Figure 5: Sample output of a fluiddynamics simulation of the $\mathrm{AV}$, showing preferential horizontal transport. Color code shows fluid-carrying capacity of each $\sim 1 \mathrm{~cm}^{2}$ mesh cell, (units: $\mathrm{kg} / \mathrm{s}$ )

\section{- Active Gradient Stabilization System (AGSS)}

A heating system stabilizes temperatures on Borexino's top boundary through a copper serpentine coupled to the top WT dome, under the TIS (monitored by LTPS' Ph.III.a). It is expected to reduce temperature gradient oscillations, avoiding the largest seasonal $\Delta \mathrm{T}$ 's which could trigger strong convection through inward-propagating stratification inversions. It also minimizes the global effects of detector cooling caused by heat loss through the base $\left(\sim 7^{\circ} \mathrm{C}\right)$. 


\section{Borexino's Computational Fluid-Dynamical (CFD) simulations}

Fluid dynamics inside Borexino (especially in its AV) cannot be directly measured beyond its temperature-derived pseudo-stable, positively stratified condition given by the prominent topbottom temperature gradient. Nevertheless, they are key to reliably understanding the background shifts correlated with temperature excursions (see Section 4) and, in particular, the mechanism behind ${ }^{210} \mathrm{Bi}^{2}{ }^{210} \mathrm{Po}$ shifts. For this reason, 2D and 3D CFD models using ELUENT ${ }^{T M}$ [13] and fed LTPS data as boundary conditions have been developed.

They determined several key parameters of Borexino's fluid dynamics, such as: (i) conductiondominated phenomena in whole-detector scales (long-term cooldown due to the TIS, seasonal change extent and speeds with and without insulation, influence of structural heatpaths, boundary effects and power exchange budgeting, etc.); (ii) AGSS influence and safe operational range; (iii) benchmarks of convection conditions by reproducing representative literature scenarios with similar Rayleigh numbers as in Borexino, as well as a thermal transport benchmark model bounded by Ph.I.b data checked through comparison with Ph.I.a temperatures in the same OB simulated positions. These benchmarks allowed to determine (iv) the leading mechanisms of fluid transport inside Borexino's AV, using a finely-meshed, simple convective model bounded by IV temperatures projected from LTPS' OB data. Challenges to this approach mainly arise, ironically, from Borexino's pseudo-stable fluid condition (long timescales $\mathscr{O}$ (months) over large dimensions $\mathscr{O}(\mathrm{m})$ ), where identifying the model's temporal and spatial granularities is paramount in order to avoid numerical biases overwhelming physical effects

It was found that global transport phenomena are horizontally-dominated, with North-South lateral $\Delta \mathrm{T}$ asymmetries in isotherms are found to dominate the fluid flow in the AV (see Figure 5). A consistent relationship between the observed simulated currents and the expected ${ }^{210} \mathrm{Bi}$-Po halflives constraint (see Section 4) was verified by displacements $\sim \mathscr{O}(\mathrm{cm}-\mathrm{m}) /$ day, validating prior hypotheses. Also, increased top-bottom gradient is seen to limit vertical circulation cell size (if the cells are seen as "pancakes", their thickness) and, with it, the extent of vertical fluid migration inside the bulk of the AV [12].

Current work focuses on clarifying fluid-dynamics' role in ${ }^{210} \mathrm{Po}$ migration, including understanding the role of surface currents as ${ }^{210} \mathrm{Bi}$ reservoirs. Forecasts of probable background distributions in the future are also being actively researched, by quantitatively relating regional polonium concentration to the fluid's local carrying capacity (see Figure 5).

\section{Borexino and the best model-independent limit for $\mu_{v}$}

The prediction of a magnetic moment for the neutrino $\left(\mu_{v}\right)$ is embedded in the Standard Model (SM) when accounting for neutrino masses, and is proportional to those. Not knowing the absolute value of any neutrino mass, there are however limits on their combined masses that place an upper limit in said $\mu_{v}$ of $\leq 10^{-18} \mu_{B}$. Current experimental limits are far (about 6 orders of magnitude) from that, and most best limits utilize strong astrophysical constraints for their predictions [15]. The best direct experimental limits come from reactor antineutrino experiments [16], and are at least an order of magnitude away from their astrophysical counterparts. Borexino is sensitive to neutrino electromagnetic effects due to the change to the neutrino-to-electron elastic 
scattering cross sections a $\mu_{v}$ would entail, being that interaction the main detection channel in the observatory. Neutrino mixing means an effective neutrino magnetic moment $\left(\mu_{v}^{e f f}\right)$ is measured in Borexino. Moreover, since EM interactions such as $v-e$ scattering are helicity flipping, there is no interference term with the electroweak interaction - which makes Borexino's detection channel the most sensitive available, boosted by the detector's low energy threshold.

${ }^{7} \mathrm{Be}$ spectral changes are the main reason a spectral fit such as the one Borexino is able to precisely perform is most sensitive to $\mu_{v}^{e f f}$. There is an important effect in the $p p$ neutrino flux as well, but is partly compensated by an opposite-sign decrease in flux. Constraints from radiochemical integral counting experiments can be employed in order to apply an additional constraint in the fit, also taking into account these radiochemical techniques are not sensitive to magnetic moments.

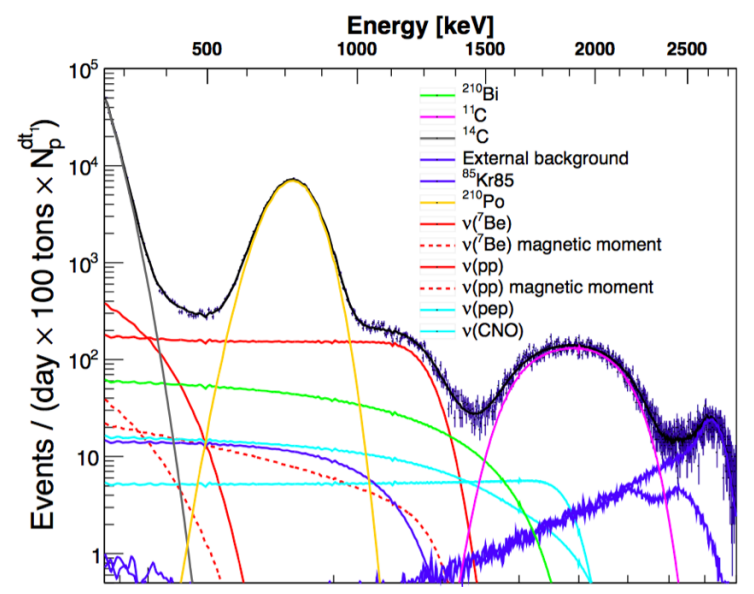

Figure 6: Borexino solar neutrino (and irreducible background) spectrum fitted to the signal of the different species, with the expected contribution of a possible neutrino magnetic moment in the $p p$ and ${ }^{7} \mathrm{Be}$ spectral components. $N_{p}^{d t_{1}}$ is an analysis variable whose correspondence to energy is shown in the upper axis.

Taking these radiochemical constraints into account, and performing a likelihood profiling of [186,2970] keV spectral fits with 15 free parameters ( 2 for resolution, one for light yield and energy scale, and 12 for signal and background species in the region of interest) over 1291.5 days of Phase 2 data the limit achieved by Borexino is $\mu_{v}^{e f f}<2.8 \cdot 10^{-11} \mu_{B}$ (90\% c.l.), which translates into $\mu_{v}^{e f f}<4.0 \cdot 10^{-11} \mu_{B}(90 \%$ c.l.) if no independent constraint on the $p p$ flux is employed. This represents the best model-independent $\mu_{v}^{e f f}$ limit available. Limits for each neutrino species can also be derived, considering the survival probability for $p p$ and ${ }^{7} \mathrm{Be}$ neutrinos is the same for both $(0.55)$ and taking the most conservative mass hierarchy choice (unfavorably to Borexino's sensitivity, the inverted one): $\mu_{v_{e}}<3.9 \cdot 10^{-11} \mu_{B}$ and $\mu_{v_{\mu / \tau}}<5.8 \cdot 10^{-11} \mu_{B}$, in all cases at $90 \%$ c.l.

\section{Conclusions and perspectives}

Borexino has performed the first simultaneous, wideband, precision solar neutrino spectroscopy of the dominant species in the solar neutrino flux (pp, pep, ${ }^{7} \mathrm{Be},{ }^{8} \mathrm{~B}$ ) with uncertainty levels as low as $2.7 \%$ (for ${ }^{7} \mathrm{Be}$ ), as well as an upper limit for $\mathrm{CNO}$ neutrinos consistent with the previous, worldbest one obtained with Borexino Phase 1 data, but using less stringent assumptions. This result, apart from confirming the SSM understanding of solar fusion to within uncertainty (and providing 
a slight hint toward the validity of the high metallicity SSMs), further strengthens the MSW-LMA parametrization of neutrino oscillations thanks to the unique probe into the vacuum-dominated survival probability region, as well as the closest available datapoints (pep and ${ }^{8} \mathrm{~B}$ 's Low Energy Region) to the transition region between 1 and $10 \mathrm{MeV}$. Additionally, Borexino has succeeded in providing the best available model-independent limit on the effective magnetic moment of the neutrino $\mu_{v}^{\text {eff }}<2.8 \cdot 10^{-11} \mu_{B}$. Thanks to the unprecedented and steadily improving radiopurity levels achieved during construction, purification and undisturbed operation, the detector went through a major upgrade focusing on thermal monitoring, insulation and management aimed at better stabilizing its Inner Volume and, consequently, minimizing scintillator mixing that could bring elevated background levels to the innermost Fiducial Volume, in order to acquire the highest-quality dataset possible. This may open avenues for further precision improvements in the already-measured solar neutrino species, or even the first direct determination of as-of-yet unmeasured neutrino fluxes, like $\mathrm{CNO}$ neutrinos. In this work, the preliminary joint fit from $p p$ to ${ }^{8} \mathrm{~B}$ neutrinos is presented in the HQL2018 conference; the detailed results and techniques will be available soon in a dedicated publication.

\section{Appendix: Borexino Collaboration}

** M. Agostini ${ }^{m}$, K. Altenmüller ${ }^{m}$, S. Appel ${ }^{m}$, V. Atroshchenko ${ }^{g}$, Z. Bagdasarian ${ }^{x}$, D. Basilico ${ }^{h}$, G. Bellini ${ }^{h}$, J. Benziger $^{k}$,

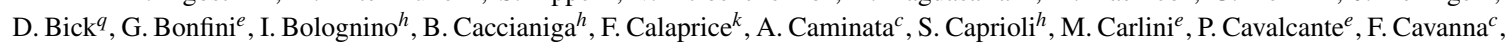

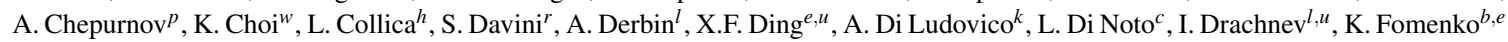
A. Formozov ${ }^{h, b, p}$, D. Franco ${ }^{a}$, F. Gabriele ${ }^{e}$, C. Galbiati ${ }^{k}$, C. Ghiano ${ }^{a}$, M. Giammarchi ${ }^{h}$, A. Goretti ${ }^{k}$, M. Gschwender ${ }^{t}$, M. Gromov $^{p}$, D. Guffanti ${ }^{e}, u$, C. Hagner ${ }^{q}$, T. Houdy ${ }^{a}$, E. Hungerford ${ }^{r}$, Aldo Ianni ${ }^{e}$, Andrea Ianni $^{k}$, A. Jany ${ }^{d}$, D. Jeschke ${ }^{m}$, V. Kobychev ${ }^{f}$, D. Korablev ${ }^{b}$, G. Korga ${ }^{r}$, T. Lachenmaier ${ }^{t}$, M. Laubenstein ${ }^{e}$, E. Litvinovich ${ }^{g}$, F. Lombardi ${ }^{e}$, P. Lombardi ${ }^{1 h}$, L. Ludhova ${ }^{i, x}$, G. Lukyanchenko ${ }^{g}$, L. Lukyanchenko ${ }^{g}$, I. Machulin ${ }^{g}$, G. Manuzio ${ }^{c}$, S. Marcocci ${ }^{2 u}$, J. Maricic ${ }^{w}$, J. Martyny ${ }^{y}$, E. Meroni ${ }^{h}$, M. Meyer ${ }^{v}$, L. Miramonti ${ }^{h}$, M. Misiaszek ${ }^{d}$, V. Muratova ${ }^{l}$, B. Neumair ${ }^{m}$, L. Oberauer ${ }^{m}$, M. Obolensky ${ }^{a}$, B. Opitz ${ }^{q}$, V. Orekhov ${ }^{g}$, F. Ortica ${ }^{j}$, M. Pallavicini $^{c}$, L. Papp ${ }^{o}$, Ö. Penek ${ }^{i, x}$, L. Pietrofaccia ${ }^{e}$, N. Pilipenko ${ }^{l}$, A. Pocar $^{n}$, A. Porcelli ${ }^{y}$, G. Raikov ${ }^{g}$, G. Ranucci $^{h}$, A. Razeto ${ }^{e}$, A. Re $^{h}$, M. Redchuk $^{i, x}$, A. Romani ${ }^{j}$, N. Rossi ${ }^{3 e}$, S. Rottenanger ${ }^{t}$, S. Schönert ${ }^{m}$, D. Semenov ${ }^{l}$, M. Skorokhvatov ${ }^{g}$, O. Smirnov $^{b}$, A. Sotnikov ${ }^{b}$, L.F.F. Stokes ${ }^{e}$, Y. Suvorovige, G. Testerac ${ }^{4}$, R. Tartaglia ${ }^{e}$, J. Thurn ${ }^{v}$, M. Toropova ${ }^{g}$, E. Unzhakov ${ }^{l}$, A. Vishneva ${ }^{b}$, R.B. Vogelaar ${ }^{o}$, M. Wojcik $^{d}$, M. Wurm ${ }^{t}$, F. von Feilitzsch ${ }^{m}$, S. Weinz ${ }^{y}$, O. Zaimidoroga ${ }^{b}$, S. Zavatarelli ${ }^{c}$, K. Zuber ${ }^{v}$, G. Zuzel ${ }^{d}$.

[a] APC, Univ. Paris Diderot, CNRS/IN2P3, CEA/Irfu, Obs. de Paris Sorbonne Paris Cité, France

[b] Joint Institute for Nuclear Research, Dubna 141980, Russia

[c] Dipartimento di Fisica, Università e INFN, Genova 16146, Italy

[d] M. Smoluchowski Institute of Physics, Jagellonian University, Krakow, 30059, Poland

[e] INFN Laboratori Nazionali del Gran Sasso, Assergi 67010, Italy

[f] Kiev Institute for Nuclear Research, Kiev 06380, Ukraine

[g] NRC Kurchatov Institute, Moscow 123182, Russia

[h] Dipartimento di Fisica, Università degli Studi e INFN, Milano 20133, Italy

[i] RWTH Aachen University, 52062 Aachen, Germany

[j] Dipartimento di Chimica, Università e INFN, Perugia 06123, Italy

[k] Princeton University, Princeton, NJ 08544, USA

[1] St. Petersburg Nuclear Physics Institute, Gatchina 188350, Russia

[m] Physik Department, Technische Universität München, Garching 85747, Germany

[n] Physics Department, University of Massachusetts, Amherst MA 01003, USA

[o] Physics Department, Virginia Polytechnic Institue and State University, Blacksburg, VA 24061, USA

[p] Lomonosov Moscow State University, Institute of Nuclear Physics, Moscow 119234, Russia [q] Institut für Experimentalphysik, Universität Hamburg, Germany

\footnotetext{
${ }^{1}$ Present address: Physics Department, University of California, San Diego, CA 92093, USA

${ }^{2}$ Present address: Fermilab National Accelerator Laboratory (FNAL), Batavia, IL 60510, (USA)

${ }^{3}$ Present address: Dipartimento di Fisica, Sapienza Università di Roma e INFN, 00185 Roma, Italy

${ }^{4}$ Present address: Dipartimento di Fisica, Università degli Studi Federico II e INFN, 80126 Napoli, Italy
} 
[r] Department of Physics, University of Houston, Houston, TX 77204, USA

[s] Physics ans Astronomy Department, University of California Los Angeles (UCLA), Los Angeles, CA 90095, USA

[t] Eberhard Karls Universität Tübingen, Physikalisches Institut, auf der Morgenstelle 14, 72076 Tb̈ingen, Germany

[u] Gran Sasso Science Institute, 67100 L'Aquila, Italy

[v] Department of Physics, Technische Universität Dresden, 01062 Dresden, Germany

[w] Department of Physics and Astronomy, University of Hawaii, Honolulu, HI 96822, USA

[x] Institut für Kernphysik, Forschungszentrum Jülich, 52425 Jülich, Germany

[y] Institute of Physics and Excellence Cluster PRISMA, Johannes Gutenberg-Universität Mainz, 55099 Mainz, GermanyÊ

\section{References}

[1] Borexino Collaboration (M. Agostini et al.) Neutrinos from the primary proton-proton fusion process in the Sun - Nature 512, 7515 383-386 (2014)

[2] Borexino Collaboration (G. Bellini et al.) First evidence of pep solar neutrinos by direct detection in Borexino - PRL 108051302 (2012)

[3] Borexino Collaboration ( $G$. Bellini et al.) Precision measurement of the ${ }^{7} \mathrm{Be}$ solar neutrino interaction rate in Borexino - PRL 107141302 (2011)

[4] Borexino Collaboration (G. Bellini et al.) Measurement of the solar ${ }^{8} \mathrm{~B}$ neutrino rate with a liquid scintillator target and $3 \mathrm{MeV}$ energy threshold in the Borexino detector - PRL 82033006 (2010)

[5] Borexino Collaboration (M. Agostini et al.) First Simultaneous Precision Spectroscopy of pp, 7Be, and pep Solar Neutrinos with Borexino Phase-II - arXiv:1707.09279v2

[6] Borexino Collaboration (M. Agostini et al.) Improved measurement of $8 \mathrm{~B}$ solar neutrinos with $1.5 \mathrm{kt}$ y of Borexino exposure - arXiv:1709.00756v1

[7] Borexino Collaboration (M. Agostini et al.) Spectroscopy of geo-neutrinos from 2056 days of Borexino data - PRD 92031101 (2015)

[8] J. Bezinger The Borexino Purification System - Int.J.Mod.Phys. A29 1442002 (2014)

[9] Borexino Collaboration (G. Bellini et al.) Borexino calibrations: Hardware, Methods, and Results JINST 7 P10018 (2012)

[10] N. Vinyoles et al. A New Generation of Standard Solar Models - The Astrophysical Journal 835, 202 (2017)

[11] D. Bravo-Berguño Precision Background Stability and Response Calibration in Borexino: Prospects for Wideband, Precision Solar Neutrino Spectroscopy and BSM Neutrino Oscillometry Through a Deeper Detector Understanding - PhD Thesis, Virginia Tech

[12] D. Bravo and R. Mereu The Borexino Thermal Monitoring and Management System and simulations of the fluid-dynamics of the Borexino detector under asymmetrical, changing boundary conditions NIM-A 885, 38-53 (2018)

[13] ANSYS Inc. - ANSYS Fluent 17.0 User's Guide (2016)

[14] Borexino Collaboration (M. Agostini et al.) The Monte Carlo simulation of the Borexino detector Astroparticle Physics 97, 136-159 (2018)

[15] SNO Collaboration (Ahmad Q R et al.) Direct Evidence for Neutrino Flavor Transformation from Neutral-Current Interactions in the Sudbury Neutrino Observatory - Phys. Rev. Lett. 89011301 (2002)

[16] A.G. Beda et al.) Gemma experiment: The results of neutrino magnetic moment search - Phys. of Part. and Nuclei Lett 10, 139 (2013) 